\title{
Role of Chain Polarity on Ion and Polymer Dynamics: Molecular Volume-Based Analysis of Dielectric Constant for Polymerized Norbornene-based Ionic Liquids
}

U Hyeok Choi, ${ }^{1,2}$ Terry L. Price, Jr., ${ }^{3, \S}$ Daniel V. Schoonover, ${ }^{3, \mathscr{Q}}$ Renxuan Xie, ${ }^{4, \ngtr}$ Harry W. Gibson, ${ }^{3}$ and Ralph H. Colby ${ }^{*}$

${ }^{1}$ Department of Polymer Science and Engineering, Inha University, Incheon 22212, Korea

${ }^{2}$ Program in Environmental and Polymer Engineering, Inha University, Incheon 22212, Korea

${ }^{3}$ Department of Chemistry, Virginia Polytechnic Institute and State University, Blacksburg, Virginia 24061, United States

${ }^{4}$ Department of Materials Science and Engineering, Pennsylvania State University, University Park, PA 16802, United States

$\S$ Present address: Zestron Corp., 11285 Assett Loop, Manassas, VA 20109 USA

${ }^{\text {If }}$ Present address: Momentive Performance Materials, Friendly, WV 26146 USA

${ }^{¥}$ Present address: Materials Department, University of California, Santa Barbara, CA, 93106 USA

*Corresponding Author E-mail: rhc@ plmsc.psu.edu. 


\section{CONTENTS}

\begin{tabular}{|l|c|}
\hline \multicolumn{1}{|c|}{ TOPIC } & PAGE \\
\hline Electrode Polarization Analysis. & S3 \\
\hline $\begin{array}{l}\text { Figure S1. Dielectric response of a polynorbornene PIL to applied AC field at T } \\
=273 \mathrm{~K} \text {; dielectric constant }\left(\varepsilon^{\prime}\right) \text {, dielectric loss }\left(\varepsilon^{\prime \prime}\right), \text { loss tangent }(\tan \delta) \text {, and in- } \\
\text { phase part of conductivity }\left(\sigma^{\prime}\right) .\end{array}$ & $\mathrm{S} 3$ \\
\hline $\begin{array}{l}\text { Figure S2. Temperature dependence of ionic conductivity } \sigma_{\mathrm{DC}} \text { for norbornene } \\
\text { imidazolium-based ROMP PILs with and without the OEx linker }\end{array}$ & $\mathrm{S} 6$ \\
\hline $\begin{array}{l}\text { Figure S3. Temperature dependence of } \varepsilon_{s} \text { and } \varepsilon_{C} \text { (inset) for norbornene-based } \\
\text { ILM and its ROMP PIL. }\end{array}$ & $\mathrm{S} 7$ \\
\hline $\begin{array}{l}\text { Figure S4. Ionic conductivity divided by static dielectric constant } \sigma_{\mathrm{DC}} / \varepsilon_{s} \text { with } \\
\text { respect to inverse temperature normalized by glass transition temperature } T_{\mathrm{g}} / T \\
\text { for ROMP PILs. }\end{array}$ & $\mathrm{S} 8$ \\
\hline $\begin{array}{l}\text { Figure S5. Glass transition temperature as a function of Keesom volume overlap } \\
\text { parameter } V_{K} / V_{m} \text { for norbornene-based ILMs and PILs and (meth)acrylate ILMs } \\
\text { and PILs. }\end{array}$ & $\mathrm{S} 9$ \\
\hline References & $\mathrm{S} 10$ \\
\hline
\end{tabular}


Electrode Polarization Analysis. A physical model of electrode polarization (EP) makes it possible to separate ionic conductivity into the number density of simultaneously conducting ions $p$ and their mobility $\mu$, since $\sigma_{D C}=e p \mu$ with $e$ the elementary charge. ${ }^{1-5}$

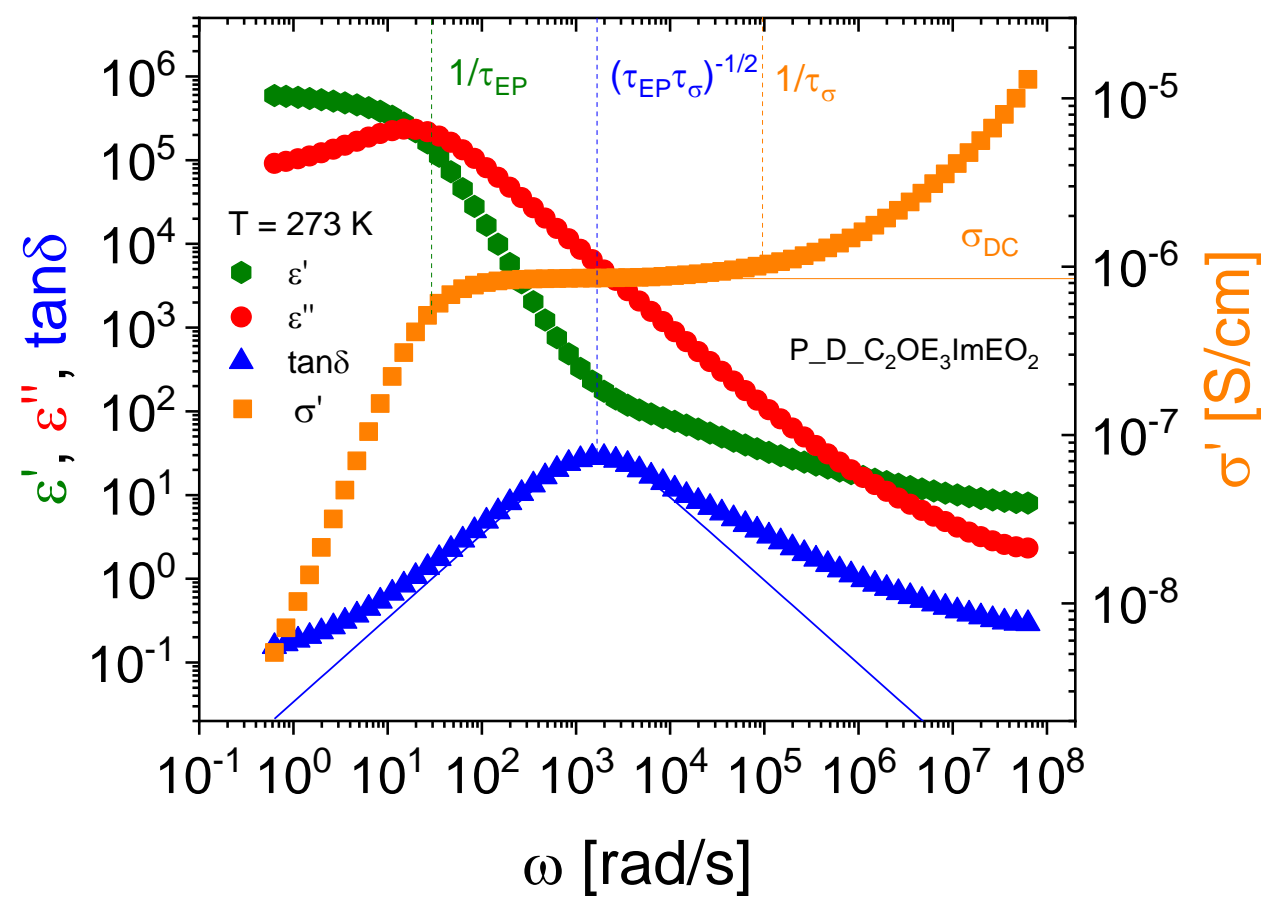

Figure S1. Dielectric response of a polynorbornene PIL (P_D_C $\left.\mathrm{C}_{2} \mathrm{OE}_{3} \mathrm{ImEO}_{2}\right)$ to applied AC field at $\mathrm{T}=273 \mathrm{~K}$; dielectric constant ( $\varepsilon^{\prime}$, green hexagons), dielectric loss ( $\varepsilon^{\prime \prime}$, red circles), loss tangent ( $\tan \delta$, blue triangles), and in-phase part of conductivity ( $\sigma^{\prime}$, orange squares). The peak of the loss tangent gives the geometric mean of the time scales of conductivity and electrode polarization $\left[\left(\tau_{\mathrm{EP}} \tau_{\sigma}\right)^{1 / 2}\right.$, dashed lines], then determining the number density of simultaneously conducting ions $p$ and their mobility $\mu$. The solid curve is a fit of $\tan \delta$ to a Debye function [ $\tan \delta=$ $\left.\omega \tau_{\mathrm{EP}} /\left(1+\omega^{2} \tau_{\sigma} \tau_{\mathrm{EP}}\right)\right]$ 
Electrode polarization occurs at low frequencies, where the transporting ions have sufficient time to polarize at the blocking electrodes during the cycle. That polarization manifests itself in (1) an increase in the effective capacitance of the cell (increasing the apparent dielectric constant) and (2) a decrease in the in-phase part of the conductivity, as the polarizing ions reduce the field experienced by the transporting ions (see Figure S1). The natural time scale for conduction is the time when counterion motion starts to become diffusive

$$
\tau_{\sigma} \equiv \frac{\varepsilon_{s} \varepsilon_{0}}{\sigma_{D C}}
$$

wherein $\varepsilon_{s}$ is the measured static relative permittivity of the sample before EP and $\varepsilon_{0}$ is the permittivity of vacuum. At low frequencies the conducting ions start to polarize at the electrodes and fully polarize at the electrode polarization time scale

$$
\tau_{E P} \equiv \frac{\varepsilon_{E P} \varepsilon_{0}}{\sigma_{D C}}
$$

wherein $\varepsilon_{E P}$ is the (considerably larger) effective permittivity after the electrode polarization is complete (see Figure S1). The Macdonald and Coelho model ${ }^{1-3,6}$ treats electrode polarization as a simple Debye relaxation with loss tangent,

$$
\tan \delta=\frac{\omega \tau_{E P}}{1+\omega^{2} \tau_{\sigma} \tau_{E P}}
$$

allowing a two-parameter fit (see solid curve in Figure S1) to determine the electrode polarization time $\tau_{E P}$ and the conductivity time $\tau_{\sigma}$. The Macdonald and Coelho model then determines the number density of simultaneously conducting ions $p$ and their mobility $\mu$ from $\tau_{E P}$ and $\tau_{\sigma}$ 


$$
\begin{aligned}
& p=\frac{1}{\pi l_{B} L^{2}}\left(\frac{\tau_{E P}}{\tau_{\sigma}}\right)^{2} \\
& \mu=\frac{e L^{2} \tau_{\sigma}}{4 \tau_{E P}^{2} k T}
\end{aligned}
$$

wherein $l_{B} \equiv e^{2} /\left(4 \pi \varepsilon_{s} \varepsilon_{0} k T\right)$ is the Bjerrum length, $L$ is the spacing between electrodes, $k$ is the Boltzmann constant and $T$ is the absolute temperature. 


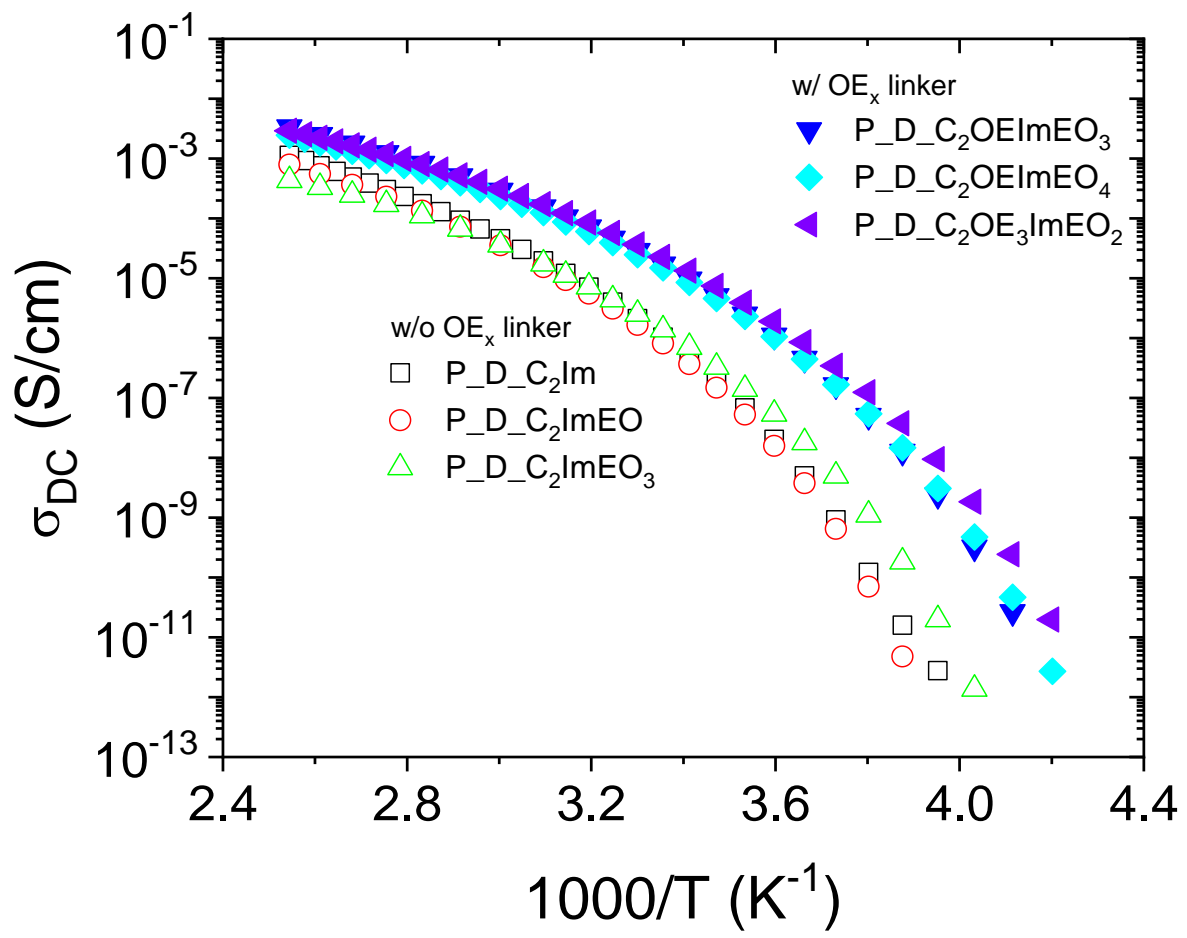

Figure S2. Temperature dependence of ionic conductivity $\sigma_{\mathrm{DC}}$ for norbornene imidazolium-based ROMP PILs with the $\mathrm{OE}_{\mathrm{x}}$ linker (filled symbols: P_D_C ${ }_{2} \mathrm{OEImEO}_{3}, \mathrm{P}_{-} \mathrm{D} \_\mathrm{C}_{2} \mathrm{OEImEO}$, and P_D_C $\mathrm{C}_{2} \mathrm{OE}_{3} \mathrm{ImEO}_{2}$ ) and without the $\mathrm{OE}$ x linker (open symbols: P_D_C $\mathrm{Im}, \mathrm{P} \_\mathrm{D} \_\mathrm{C}_{2} \mathrm{ImEO}$, and P_D_C $\mathrm{ImEO}_{3}$ ). The PILs with the $\mathrm{OE}_{\mathrm{x}}$ linker have significantly higher ionic conductivity than those without the $\mathrm{OE}_{\mathrm{x}}$ linker. 


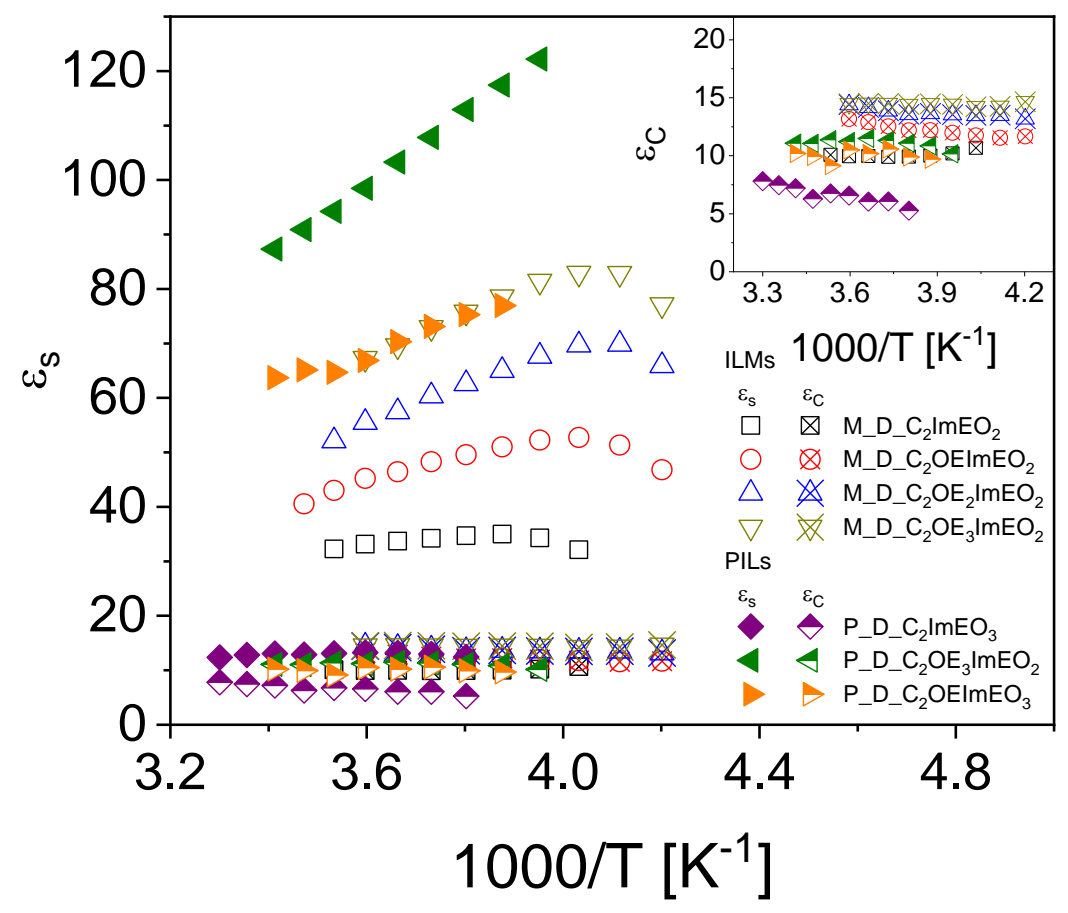

Figure S3. Temperature dependence of static dielectric constant $\varepsilon_{s}$ and Coulombic dielectric constant $\varepsilon_{C}$ (inset) for norbornene-based ionic liquid monomers (ILMs, open and X symbols) and ROMP norbornene-based polymerized ionic liquids (PILs, filled and half up symbols). The PILs have higher $\varepsilon_{s}$ than the ILMs, while the PILs have lower $\varepsilon_{C}$ than the ILMs. 

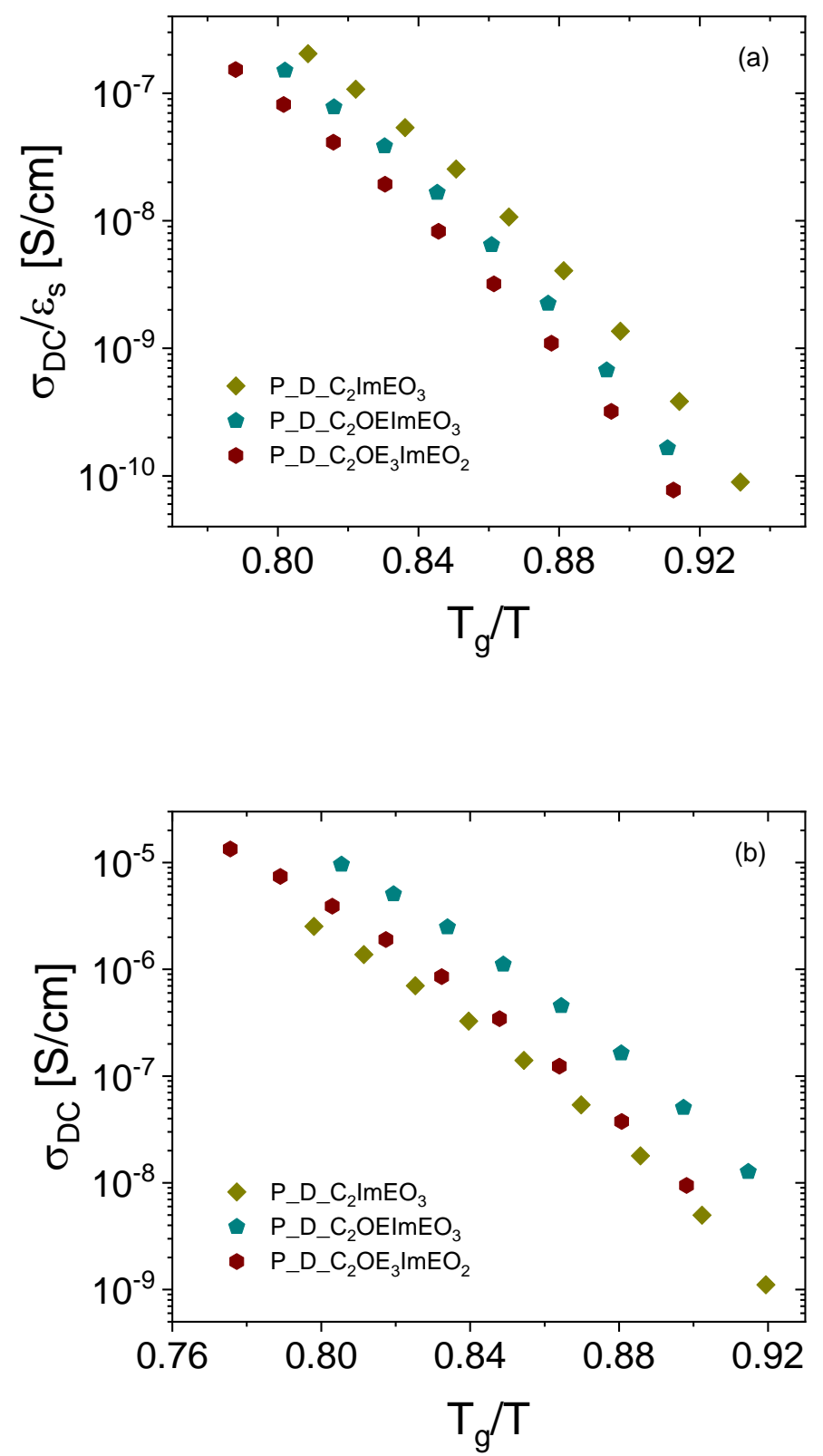

Figure S4. (a) Ionic conductivity divided by static dielectric constant $\sigma_{\mathrm{DC}} / \varepsilon_{s}$ plotted against inverse temperature normalized by glass transition temperature $T_{\mathrm{g}} / T$ for ROMP PILs. Their ionic conductivities do not merge into a single curve in this plot but they do when plotting $\sigma_{\mathrm{DC}} / \varepsilon_{c}$ vs. $T_{\mathrm{g}} / T$, see Fig. 5e of the paper. (b) Ionic conductivities with respect to inverse temperature normalized by glass transition temperature for ROMP PILs 


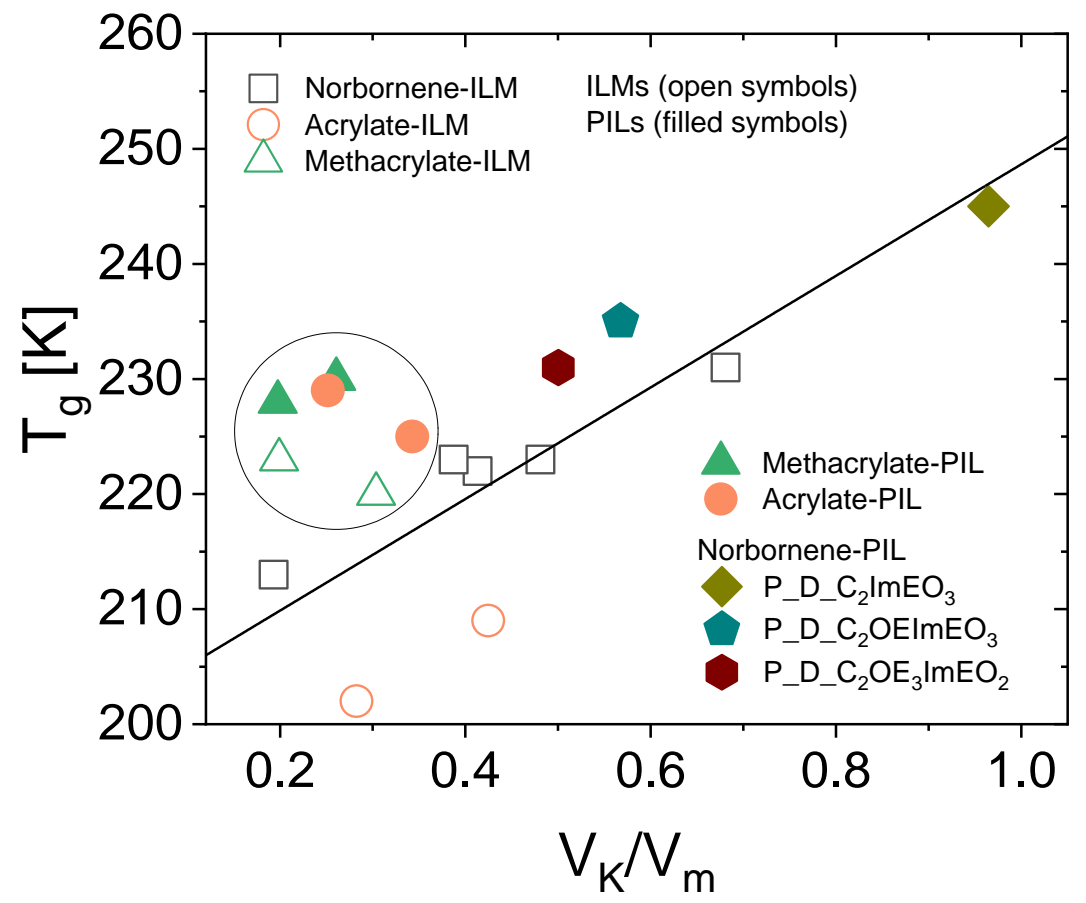

Figure S5. Glass transition temperature as a function of Keesom volume overlap parameter $V_{K} / V_{m}$ for norbornene-based ILMs ${ }^{7,8}$ and PILs ${ }^{9}$ and (meth)acrylate ILMs ${ }^{10,11}$ and PILs ${ }^{5,11}$ with a solid line as a guide for the eye. The deviation from the relation was circled for the acrylate PILs, methacrylate ILMs, and their PILs having relatively high $T_{\mathrm{g}}$. 


\section{References}

(1) Macdonald, J. R. Theory of Ac Space-Charge Polarization Effects in Photoconductors, Semiconductors, and Electrolytes. Phys. Rev. 1953, 92, 4-17.

(2) Coelho, R. Sur La Relaxation d'une Charge d'espace. Rev. Phys. Appl. 1983, 18, 137-146.

(3) Klein, R. J.; Zhang, S. H.; Dou, S.; Jones, B. H.; Colby, R. H.; Runt, J. Modeling Electrode Polarization in Dielectric Spectroscopy: Ion Mobility and Mobile Ion Concentration of Single-Ion Polymer Electrolytes. J. Chem. Phys. 2006, 124, 144903.

(4) Fragiadakis, D.; Dou, S.; Colby, R. H.; Runt, J. Molecular Mobility and $\mathrm{Li}^{+}$Conduction in Polyester Copolymer Ionomers Based on Poly(ethylene oxide). J. Chem. Phys. 2009, 130, 64907.

(5) Choi, U. H.; Lee, M.; Wang, S.; Liu, W.; Winey, K. I.; Gibson, H. W.; Colby, R. H. Ionic Conduction and Dielectric Response of Poly(imidazolium acrylate) Ionomers. Macromolecules 2012, 45, 3974-3985.

(6) Coelho, R. Physics of Dielectrics for the Engineer; Elsevier: New York, 1979.

(7) Price, T. L.; Choi, U. H.; Schoonover, D. V.; Arunachalam, M.; Xie, R.; Lyle, S.; Colby, R. H.; Gibson, H. W. Ion Conducting ROMP Monomers Based on (Oxa)Norbornenes with Pendant Imidazolium Salts Connected via Oligo(oxyethylene) Units and with Oligo(ethyleneoxy) Terminal Moieties. Macromolecules 2019, 52, 1371-1388. 
(8) Choi, U. H.; Price, T. L.; Schoonover, D. V.; Gibson, H. W.; Colby, R. H. The Effect of Oligo(oxyethylene) Moieties on Ion Conduction and Dielectric Properties of NorborneneBased Imidazolium $\mathrm{Tf}_{2} \mathrm{~N}$ Ionic Liquid Monomers. Macromolecules 2020, 53, 4990-5000.

(9) Price, T. L.; Choi, U. H.; Schoonover, D. V; Wang, D.; Heflin, J. R.; Xie, R.; Colby, R. H.; Gibson, H. W. Studies of Ion Conductance in Polymers Derived from Norbornene Imidazolium Salts Containing Ethyleneoxy Moieties. Macromolecules 2019, 52, 13891399.

(10) Lee, M.; Choi, U. H.; Colby, R. H.; Gibson, H. W. Ion Conduction in Imidazolium Acrylate Ionic Liquids and Their Polymers. Chem. Mater. 2010, 22, 5814-5822.

(11) Choi, U. H.; Mittal, A.; Price, T. L.; Gibson, H. W.; Runt, J.; Colby, R. H. Polymerized Ionic Liquids with Enhanced Static Dielectric Constants. Macromolecules 2013, 46, 11751186. 